\title{
Quantification of Cell-Free DNA: A Comparative Study of Three Different Methods
}

Kibum Jeon, Jiwon Lee, Jee-Soo Lee, Miyoung Kim, Han-Sung Kim, Hee Jung Kang, and Young Kyung Lee Department of Laboratory Medicine, Hallym University Sacred Heart Hospital, Anyang, Korea

Corresponding author: Jee-Soo Lee Department of Laboratory Medicine, Hallym University Sacred Heart Hospital, 22 Gwanpyeong-ro 170beon-gil, Dongan-gu, Anyang 14068, Korea

Tel: +82-31-380-4729

Fax: +82-31-380-1798

E-mail: jsleemd85@gmail.com

\begin{abstract}
Background: Cell-free DNA (cfDNA) provides unique potential as a biomarker for cancer patients or in the field of prenatal care. Following extraction from plasma, cfDNA should be quantified before downstream analysis. The standardization of the quantification methods is essential for application of these techniques to laboratory practice. We aimed to compare three different quantification methods (spectrophotometry, fluorometry, and the electrophoresis-based method) and to determine the contributor(s) to the differences, if any. Methods: A total of 135 plasma samples obtained from cancer patients $(n=71)$ and normal individuals $(n=15)$ and cfDNA were extracted from the samples. The extracted cfDNA was quantified using three different methods: NanoDrop spectrophotometry, Quantus fluorometry, and the electrophoresis-based 4200 TapeStation method.

Results: NanoDrop exhibited the highest estimates (median, $12.3 \mathrm{ng} / \mathrm{uL}$ ). The estimates of Quantus (median, $0.33 \mathrm{ng} / \mathrm{uL}$ ) were higher than those of 4200 TapeStation (median, $0.22 \mathrm{ng} /$ $\mathrm{uL})$. Quantus and TapeStation were significantly correlated $(r=0.802, P<0.001)$. The BlandAltman plot showed a positive bias of $0.24 \mathrm{ng} / \mathrm{uL}$ for Quantus compared with TapeStation. The difference between Quantus and TapeStation values demonstrated a significantly moderate correlation with the amount of high molecular weight DNA $(r=0.357, P<0.001)$. Conclusions: Nanodrop is considered as a non-specific method of cfDNA quantification. Quantus depicted more specific quantification compared to Nanodrop; however, TapeStation may be used in parallel or as a complementary method as it enables to determine the size distribution of DNA fragments.
\end{abstract}

(Lab Med Qual Assur 2019;41:214-219)

Key Words: Cell-free nucleic acids, Spectrophotometry, Fluorometry

Received July 2, 2019, Revision received August 8, 2019, Accepted August 22, 2019

\section{INTRODUCTION}

Cell-free DNA (cfDNA) is a subset of circulating extracellular DNA in plasma, which is released from cells mostly through apoptosis, necrosis, and active secretion 〔1]. The cfDNA is easily obtained by using minimally invasive procedures and has unique potential biomarkers for non-invasive prenatal testing and cancer management $[2,3]$. The recent advances in molecular technologies such as next-generation sequencing (NGS) and digital polymerase chain reaction (dPCR) have enabled routine analysis in clinical laboratories [4].

The standardization of the analytical conditions to detect the genetic alterations in cfDNA still remains a major challenge [5]. After isolation from plasma, cfDNA is quantified before downstream analysis (e.g., NGS and $\mathrm{dPCR}$ ). An accurate measurement of cfDNA, as a stringent quality control of cfDNA isolation, is a critical preliminary step for these downstream analyses [6] Several methods have been described to quantify cfDNA. Quantitative PCR (qPCR) has been used to measure DNA concentration by targeting various pre-selected DNA sequences, and recently more rapid and costeffective methods such as spectrophotometry, fluorometry, and electrophoresis-based methods are being used [5]. Spectrophotometers (e.g., NanoDrop) measure the 


\section{LABORATORY MEDICINE and QUALITY ASSURANCE}

Kibum Jeon et al • Methods for Quantification of cfDNA

maximal absorbance at $260 \mathrm{~nm}$. Fluorometers (e.g., Qubit and Quantus) use fluorescent probes which determine the DNA concentration. Each fluorescent probe is specifically bound to one type of molecule (double-stranded DNA [dsDNA] or single-stranded DNA [ssDNA]), which enables us to measure relatively low concentrations of cfDNA [7]. Electrophoresis-based instruments (e.g., TapeStation and Bioanalyzer) allow fluorometric quantification and visualize the concentration of cfDNA by size. However, there is lack of published evidence to characterize the differences between these methods.

The purpose of this study was to perform a headto-head comparison among the aforementioned three different quantification methods (spectrophotometry, fluorometry, and electrophoresis-based methods) and to determine the contributor to the any differences between them.

\section{MATERIALS AND METHODS}

\section{Blood collection}

A total of 135 blood samples from 71 patients (54 with colorectal cancer and 17 with pancreatic adenocarcinoma) and 15 normal individuals were collected, including 29 patients from whom multiple sequential blood samples were taken during follow-up. Blood samples (3.0 mL) were collected into BD Vacutainer $\mathrm{K}_{2}$ EDTA tubes (Becton Dickinson, Franklin Lakes, NJ, USA). After the drawing of blood, the tubes were further processed within 3 hours.

\section{Plasma preparation}

Blood samples were centrifuged at $1,600 \times \mathrm{g}$ for 10 minutes, at room temperature. A total of $1.5 \mathrm{~mL}$ of each plasma sample was transferred to $1.5 \mathrm{~mL}$ microtubes (Eppendorf, Hamburg, Germany). In order to remove any residual blood compartments, the plasma fractions were sequentially centrifuged at $13,000 \times \mathrm{g}$ for 10 minutes. The supernatant was transferred to clean $1.5 \mathrm{~mL}$ microtubes and stored at $-80^{\circ} \mathrm{C}$ until further analysis.

\section{DNA isolation and quantification}

cfDNA was manually extracted from plasma $(1 \mathrm{~mL})$ using a QIAmp circulating nucleic acid kit (Qiagen, Hilden, Germany), according to the manufacturer's instructions. The cfDNA was eluted in $50 \mathrm{uL}$ Buffer AVE provided by Qiagen and stored at $4^{\circ} \mathrm{C}$, until further analysis. The isolated cfDNA was quantified using three different methods: NanoDrop ND-2000 spectrophotometer (Nanodrop Technologies, Wilmington, DE, USA), Quantus fluorometer with the QuantiFluor ONE dsDNA kit for a specific quantification of dsDNA (Promega, Madison, WI, USA), and the microfluidic platform-based electrophoresis 4200 TapeStation instrument with high sensitivity D1000 Tape and reagents that enable the determination of the distribution of DNA fragments ranged between 50 and 1,000 bp (Agilent, Santa Clara, CA, USA). All the protocols followed the manufacturers' instructions.

\section{Statistical analysis}

The statistical differences in DNA concentration were tested using Friedman test, Wilcoxon signed rank test, and Mann-Whitney U-test. Pearson correlation and Bland-Altman plot were used to assess the correlation and differences between Quantus and TapeStation. $P$-values $<0.05$ were considered statistically significant.

\section{RESULTS}

The concentrations of isolated cfDNA were estimated using three different quantification methods (NanoDrop spectrophotometry, Quantus fluorometry, and the electrophoresis-based 4200 TapeStation method). The concentration estimates differed significantly among the different methods $(P<0.001)$ (Fig. 1$)$. The NanoDrop exhibited the highest estimates (median, $12.30 \mathrm{ng} /$ uL; range, 9.5-23.9 ng/uL). The estimates of the Quantus fluorometer were higher than those of the 4200 TapeStation (Quantus: median, $0.33 \mathrm{ng} / \mathrm{uL}$; range, 0.0714.420 ng/uL; TapeStation: median, $0.22 \mathrm{ng} / \mathrm{uL}$; range, 0.039-4.040 ng/uL).

The estimates of Quantus and TapeStation were significantly higher in cancer patients as compared to those of the healthy individuals (Quantus: median, $0.379 \mathrm{ng} /$ uL versus 0.236 ng/uL; $P=0.005$; TapeStation: median, 


\section{LABORATORY MEDICINE and QUALITY ASSURANCE}

Kibum Jeon et al Methods for Quantification of cfDNA

$0.224 \mathrm{ng} / \mathrm{uL}$ versus $0.143 \mathrm{ng} / \mathrm{uL} ; P=0.015$ ). However, the NanoDrop values of cancer patients did not significantly differ from those of the healthy individuals $(P=0.191)$ (Fig. 2). The agreement between the two methods, Quantus and TapeStation, was then assessed. These two quantification methods were found to be significantly correlated $(r=0.802$, $P<0.001$ ) (Fig. 3A). The Bland-Altman plot showed a positive bias of $0.24 \mathrm{ng} / \mathrm{uL}$ for Quantus when compared with TapeStation (Fig. 3B). Interestingly, we observed that high molecular weight (HMW) DNA (>800 bp) was

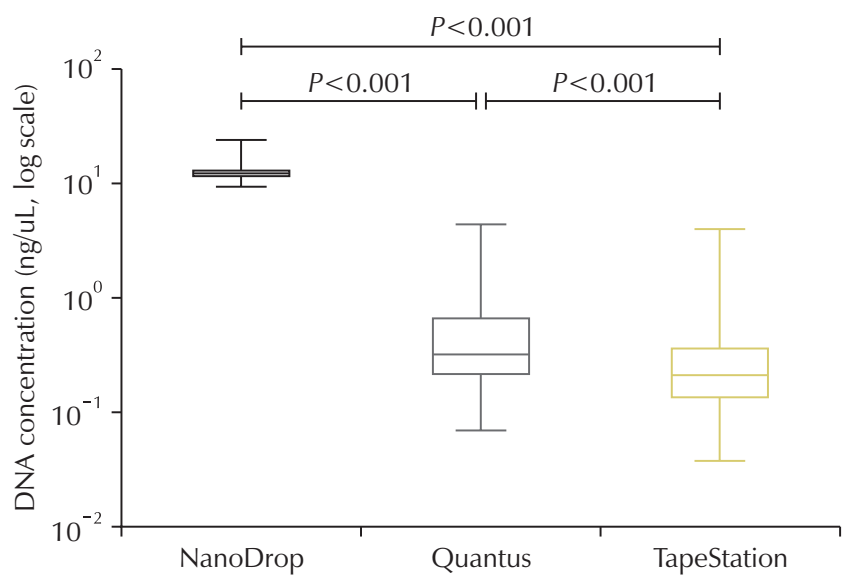

Fig. 1. Concentration estimates obtained using three different quantification methods, NanoDrop, Quantus, and TapeStation. The line within the box corresponds to the median. The lower limits and upper limits of the box indicate 0.25 and 0.75 quartiles, respectively.

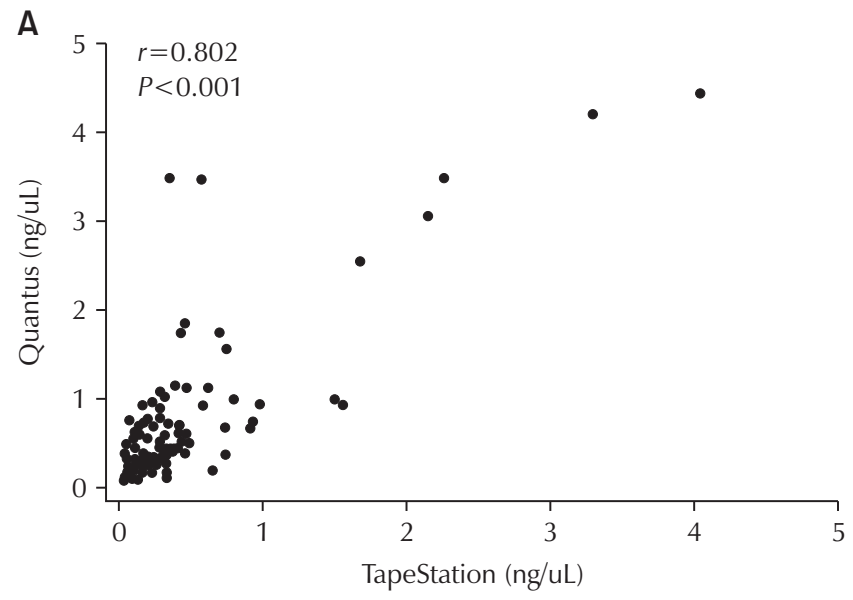

present on the electropherogram of TapeStation (Fig. 4A). It is noteworthy that the difference between Quantus and TapeStation demonstrated a significantly moderate correlation with the amount of HMW DNA $(r=0.357, P<$ 0.001) (Fig. 4B).

\section{DISCUSSION}

Accurate and easily applicable strategies of cfDNA quantification are needed for cfDNA analysis. In the current study, we characterized the three different cfDNA

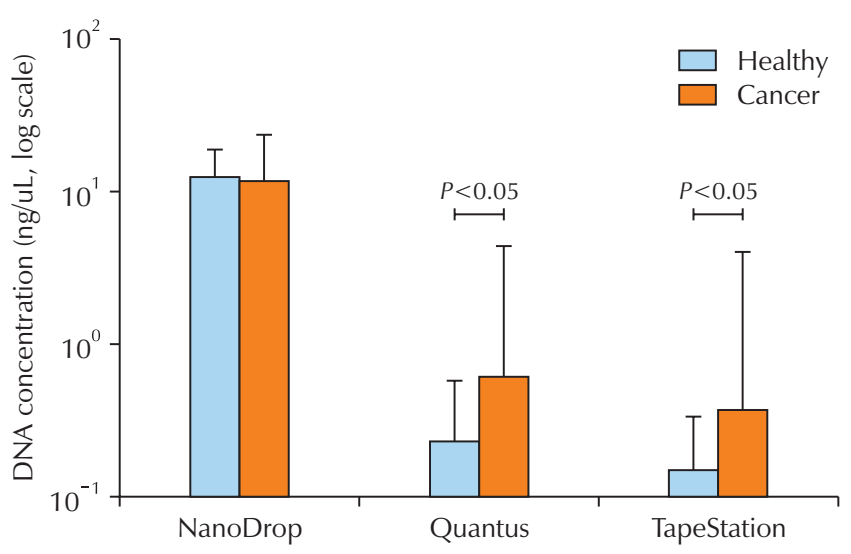

Fig. 2. Concentration estimates obtained using three different quantification methods, NanoDrop, Quantus and TapeStation. The white bars represent the results from healthy individuals, and grey bars represent the results from cancer patients.

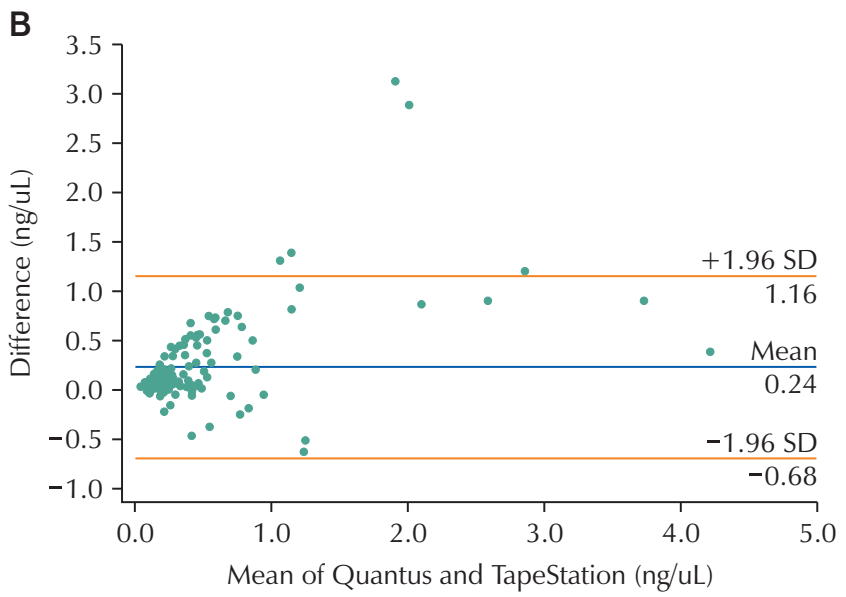

Fig. 3. (A) Scatter plot and (B) Bland-Altman for Quantus and TapeStation. Abbreviation: SD, standard deviation. 


\section{LABORATORY MEDICINE and QUALITY ASSURANCE}

Kibum Jeon et al • Methods for Quantification of cfDNA
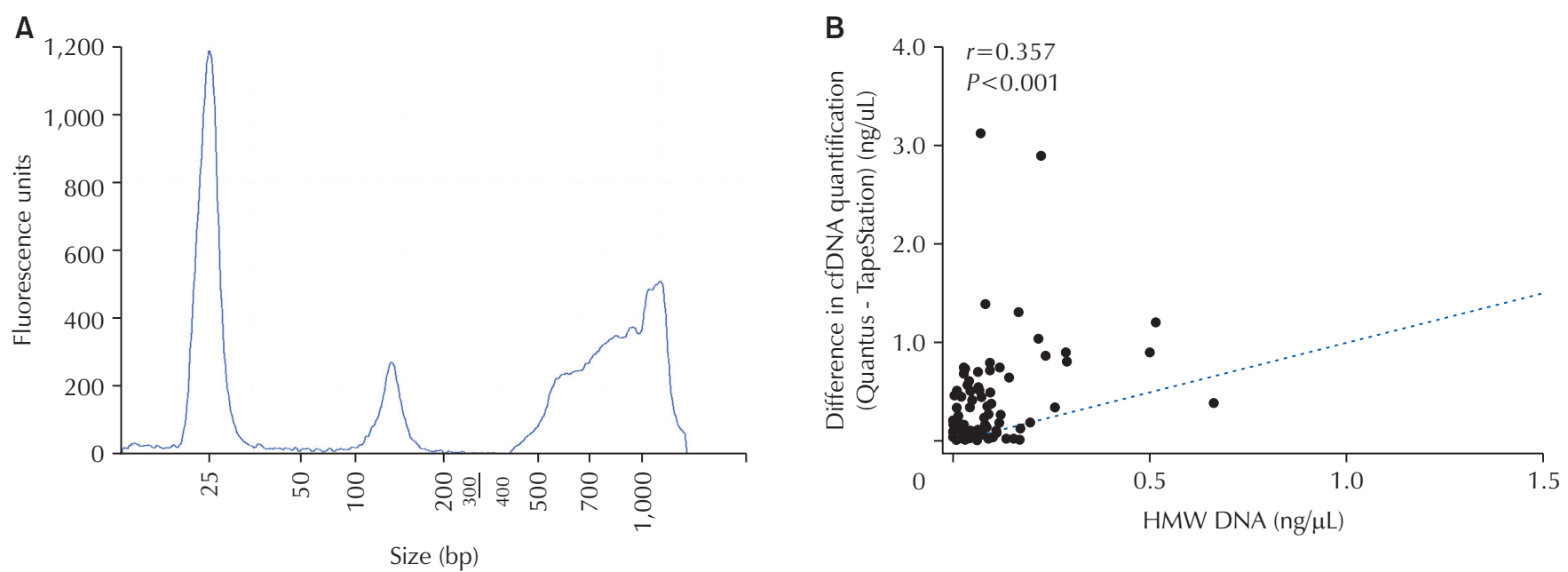

Fig. 4. (A) Electropherogram of cfDNA samples analyzed with high sensitivity D1000 tape of TapeStation. The distinct peak between around 170 bp represents the mono-nucleosomal fragment. The broad peak larger than 800 bp represents HMW DNA. (B) The difference in cfDNA quantification correlated with the amount of HMW DNA (ng/ $\mu \mathrm{L})$. Abbreviations: cfDNA, cell-free DNA; HMW, high molecular weight.

quantification methods using clinical samples. Our results showed that each method provides a different estimate of cfDNA concentration, which may be attributed to the different quantification strategies employed by each of these methods.

As expected, NanoDrop reported significantly higher concentration estimates than Quantus and TapeStation. NanoDrop estimates could not efficiently differentiate the cancer patients from healthy individuals, while those from the other two methods could do so. The high estimates of this spectrophotometric method may have occurred because of its inability to distinguish between dsDNA and ssDNA, between cfDNA fragments and protein-DNA complexes. The contaminants that absorb light at a wavelength similar to that of cfDNA may be also responsible for the overestimation by the spectrophotometer [8,9].

Quantus exhibited concentration estimates correlated to, but higher than, those of TapeStation. The difference between the two methods increased as the amount of HMW DNA increased. In this study, the DNA fragments larger than $800 \mathrm{bp}$ were classified as HMW DNA $[4,10]$. The cf DNA presents a major peak at 166 bp corresponding to nucleosomal DNA, and the size of DNA ranges from $166 \mathrm{bp}$ to approximately $500 \mathrm{bp}$ according to the mono-, di-, and tri- nucleosome structure $[4,11,12]$. Thus, the longer DNA fragments were considered as genomic DNA (gDNA) mostly likely originated from lysed hematopoietic cells $[13,14]$. Measurement of cfDNA by Quantus is based on the fluorescence intensity of fluorescent dye that directly binds to dsDNA [7]. It is important to note that the Quantus device is not able to differentiate between the size of DNA fragments [15]. On the other hand, TapeStation enables visual assessment of the distribution of fragment sizes and measures the nucleic acid concentrations for the selected size: This method has been applied in cfDNA analyses and helped to visualize the amount of distinct mono and di nucleosome DNA fragments [16-18].

Fluorometric quantification provides easy workflow and is less time consuming as compared to qPCR, therefore, a fluorometer (e.g., Qubit and Quantus) is preferred in clinical laboratories [5]. Using fluorescent probes that directly bind to dsDNA, the fluorometric quantification is an approach that compensates for the disadvantage of the spectrophotometry-based methods (e.g., NanoDrop) [19]. Several previous studies have described that the fluorometric quantification is highly correlated to $\mathrm{PPCR}$ quantification measurements and APCR results 〔2022]. The fragment lengths of isolated cfDNA usually are 


\section{LABORATORY MEDICINE and QUALITY ASSURANCE}

Kibum Jeon et al • Methods for Quantification of cfDNA

approximately $166 \mathrm{bp}$ in size, corresponding to mononucleosomal DNA. However, high molecular gDNA contamination is often observed in cfDNA isolation, which can be distinguished by electrophoresis-based methods only. The use of the electrophoresis-based method in parallel with fluorometric methods is highly recommended when quantification along with cfDNA sizing is required for NGS, as a downstream analysis $[5,15,23]$. The electrophoresis-based methods may also be used to assess the quality of the isolated cfDNA prior to downstream analysis, when the preanalytical conditions such as sample storage and shipping seem to be susceptible to gDNA contamination from cell lysis [24,25].

A major limitation of this study is that the reproducibility of each platform could not be demonstrated as we were not able to perform each experiment more than once. Further studies are required to investigate the analytical performance of each method. We believe that both fluorometric and electrophoresis based approaches may be used as parallel or complementary methods as mentioned above, and these technologies will influence the standardization of measuring cfDNA concentration.

\section{ACKNOWLEDGMENTS}

This work was supported by the Korean Association of External Quality Assessment Service (KEQAS) Research Fund of 2018 (2018-15).

\section{REFERENCES}

1. Wan JC, Massie C, Garcia-Corbacho J, Mouliere F, Brenton JD, Caldas C, et al. Liquid biopsies come of age: towards implementation of circulating tumour DNA. Nat Rev Cancer 2017;17:223-38.

2. Merker JD, Oxnard GR, Compton C, Diehn M, Hurley P, Lazar AJ, et al. Circulating tumor DNA analysis in patients with cancer: American Society of Clinical Oncology and College of American Pathologists Joint Review. J Clin Oncol 2018;36:1631-41.

3. Advani HV, Barrett AN, Evans MI, Choolani M. Challenges in non-invasive prenatal screening for sub-chro- mosomal copy number variations using cell-free DNA. Prenat Diagn 2017;37:1067-75.

4. Heitzer E, Ulz P, Geigl JB. Circulating tumor DNA as a liquid biopsy for cancer. Clin Chem 2015;61:112-23.

5. Meddeb R, Pisareva E, Thierry AR. Guidelines for the preanalytical conditions for analyzing circulating cell-free DNA. Clin Chem 2019;65:623-33.

6. Trigg RM, Martinson LJ, Parpart-Li S, Shaw JA. Factors that influence quality and yield of circulating-free DNA: a systematic review of the methodology literature. Heliyon 2018;4:e00699.

7. Nakayama Y, Yamaguchi H, Einaga N, Esumi M. Pitfalls of DNA quantification using DNA-binding fluorescent dyes and suggested solutions. PLoS One 2016;11:e0150528.

8. Jorgez CJ, Dang DD, Simpson JL, Lewis DE, Bischoff FZ. Quantity versus quality: optimal methods for cell-free DNA isolation from plasma of pregnant women. Genet Med 2006;8:615-9.

9. Goldshtein H, Hausmann MJ, Douvdevani A. A rapid direct fluorescent assay for cell-free DNA quantification in biological fluids. Ann Clin Biochem 2009;46(Pt 6):48894.

10. Fernando MR, Jiang C, Krzyzanowski GD, Ryan WL. Analysis of human blood plasma cell-free DNA fragment size distribution using EvaGreen chemistry based droplet digital PCR assays. Clin Chim Acta 2018;483:39-47.

11. Heitzer E, Speicher MR. One size does not fit all: sizebased plasma DNA diagnostics. Sci Transl Med 2018;10: eaav3873.

12. Chandrananda D, Thorne NP, Bahlo M. High-resolution characterization of sequence signatures due to nonrandom cleavage of cell-free DNA. BMC Med Genomics 2015;8:29.

13. Ulz P, Auer M, Heitzer E. Detection of circulating tumor DNA in the blood of cancer patients: an important tool in cancer chemoprevention. Methods Mol Biol 2016;1379:45-68.

14. Parpart-Li S, Bartlett B, Popoli M, Adleff V, Tucker L, Steinberg R, et al. The effect of preservative and temperature on the analysis of circulating tumor DNA. Clin Cancer Res 2017;23:2471-7. 


\section{LABORATORY MEDICINE and QUALITY ASSURANCE}

Kibum Jeon et al $\bullet$ Methods for Quantification of cfDNA

15. Hussing C, Kampmann ML, Mogensen HS, Borsting C, Morling N. Quantification of massively parallel sequencing libraries: a comparative study of eight methods. Sci Rep 2018;8:1110.

16. Lee JS, Hur JY, Kim IA, Kim HJ, Choi CM, Lee JC, et al. Liquid biopsy using the supernatant of a pleural effusion for EGFR genotyping in pulmonary adenocarcinoma patients: a comparison between cell-free DNA and extracellular vesicle-derived DNA. BMC Cancer 2018;18:1236.

17. Streleckiene G, Reid HM, Arnold N, Bauerschlag D, Forster M. Quantifying cell free DNA in urine: comparison between commercial kits, impact of gender and interindividual variation. Biotechniques 2018;64:225-30.

18. Hellwig S, Nix DA, Gligorich KM, O'Shea JM, Thomas A, Fuertes CL, et al. Automated size selection for short cellfree DNA fragments enriches for circulating tumor DNA and improves error correction during next generation sequencing. PLoS One 2018;13:e0197333.

19. Sarnecka AK, Nawrat D, Piwowar M, Ligeza J, Swadzba J, Wojcik P. DNA extraction from FFPE tissue samples: a comparison of three procedures. Contemp Oncol (Pozn) 2019;23:52-8.

20. Van Ginkel JH, van den Broek DA, van Kuik J, Linders D, de Weger R, Willems SM, et al. Preanalytical blood sample workup for cell-free DNA analysis using Drop- let Digital PCR for future molecular cancer diagnostics. Cancer Med 2017;6:2297-307.

21. Ramachandran K, Speer CG, Fiddy S, Reis IM, Singal R. Free circulating DNA as a biomarker of prostate cancer: comparison of quantitation methods. Anticancer Res 2013;33:4521-9.

22. Garcia J, Dusserre E, Cheynet V, Bringuier PP, BrenglePesce K, Wozny AS, et al. Evaluation of pre-analytical conditions and comparison of the performance of several digital PCR assays for the detection of major EGFR mutations in circulating DNA from non-small cell lung cancers: the CIRCAN_0 study. Oncotarget 2017;8:87980-96.

23. Lai J, Du B, Wang Y, Wu R, Yu Z. Next-generation sequencing of circulating tumor DNA for detection of gene mutations in lung cancer: implications for precision treatment. Onco Targets Ther 2018;11:9111-6.

24. Van Dessel LF, Vitale SR, Helmijr JC, Wilting SM, van der Vlugt-Daane M, Oomen-de Hoop E, et al. High-throughput isolation of circulating tumor DNA: a comparison of automated platforms. Mol Oncol 2019;13:392-402.

25. Van Dessel LF, Beije N, Helmijr JC, Vitale SR, Kraan J, Look MP, et al. Application of circulating tumor DNA in prospective clinical oncology trials: standardization of preanalytical conditions. Mol Oncol 2017;11:295-304. 